\title{
Fabry disease: what the cardiologist should consider in non-cardiac screening, diagnosis, and management-narrative review
}

\author{
Claudia Regenbogen, Matthias Christoph Braunisch, Christoph Schmaderer, Uwe Heemann \\ Department of Nephrology, Technical University of Munich, School of Medicine, Klinikum rechts der Isar, Munich, Germany \\ Contributions: (I) Conception and design: C Regenbogen, U Heemann; (II) Administrative support: MC Braunisch, C Schmaderer; (III) Provision of \\ study materials or patients: None; (IV) Collection and assembly of data: None; (V) Data analysis and interpretation: None; (VI) Manuscript writing: \\ All authors; (VII) Final approval of manuscript: All authors. \\ Correspondence to: Claudia Regenbogen. Department of Nephrology, Technical University of Munich, School of Medicine, Klinikum rechts der Isar, \\ Ismaningerstraße 22, 81675 Munich, Germany. Email: Claudia.Regenbogen@mri.tum.de.
}

\begin{abstract}
Fabry disease (FD) is a rare X chromosomally transmitted lysosomal storage disorders with an absence or deficiency of the enzyme alpha-galactosidase. The deposition of globotriaosylceramide (Gb3) may cause damage to all organs, particularly brain, heart and kidney. While acroparaesthesia, hypo- or anhydrosis and diarrhoea are the main symptoms in childhood, cardiac involvement with left ventricular hypertrophy (LVH), renal insufficiency, diffuse pain attacks and apoplexy are the main symptoms in adulthood. Regular examinations are necessary to record organ involvement and its progression. A major challenge is therefore to make a diagnosis at an early disease stage. This is the only way that treatment can be started if there is an indication. If FD is suspected, alpha-galactosidase should be tested in male patients and genetic testing should be performed in females to confirm the diagnosis. Since 2001, enzyme replacement therapy (ERT) has been available as a causal therapy. In 2016, chaperone therapy with the drug Migalastat was approved in the European Union, which leads to stabilisation of the defective alpha-galactosidase. Studies on gene therapy to cure FD in phase I/II. This review summarizes which patient should be screened, how to confirm the diagnosis and which examinations should be performed in FD patients during the course of the disease.
\end{abstract}

Keywords: Fabry disease (FD); lysosomal storage disorders; lyso-globotriaosylceramide (Gb3); organ involvement in Fabry disease; management

Submitted Oct 08, 2020. Accepted for publication Feb 05, 2021.

doi: $10.21037 / \mathrm{cdt}-20-845$

View this article at: http://dx.doi.org/10.21037/cdt-20-845

\section{Introduction}

Fabry's disease (FD) was independently described by Johannes Fabry and William Anderson in 1898 (1). FD is a chronically progressive disease that reduces life expectancy and restricts quality of life.

The underlying cause of FD is a lysosomal storage defect. $\mathrm{FD}$ is a rare disease with reported prevalence ranging from $1 / 40,000$ to $1 / 117,000$ (2-4). However, higher prevalence have been described in newborn screening $(1 / 3,100$ to $1 / 8,454)$ suggesting an overall higher prevalence (5). In the newborn screening, all types of variants on the GLA gene were included in the analysis, including variants of uncertain significance. This is most likely the reason for the high prevalence (6).

FD is $x$-chromosomal transmitted and can affect all organ systems of the body. The defect encoding the enzyme galactosidase A (GLA) is located in the region q21, 22 in the long arm of the $\mathrm{X}$ chromosome. To date, more than 900 mutations of the GLA gen have been detected (7), among them many of unclear significance (GUVS) (8).

The mutations in the GLA gene reduce or deteriorate the function of alpha-GLA. This results in an accumulation 
of globotriaosylceramide (Gb3) in the lysosomes of the organs, including the endothelial cells of the vessel walls (9).

The differentiation into a classic phenotype and a late phenotype is used to differentiate the course of the disease and the prognosis (10). The heterozygous classic phenotype is characterised by an undetectable or significantly reduced GLA $(<3 \%)$ as well as an early occurrence of symptoms of several organs. In the classic phenotype, organ damage can be detected at an age of 20 to 30 years.

The hemizygous late phenotype differs from the classic type as GLA is only slightly reduced and organ damage occurs much later (around the age of 50), whereby usually only one organ is affected (10).

Particularly affected by the accumulation of Gb3 are kidneys, heart, vessels and the nervous system (11). Depending on the course of the disease, the accumulation of $\mathrm{Gb3}$ can induce organ damage of varying degrees. Due to the extremely varying symptoms, the diagnosis is very often seriously delayed.

Recent publications have shown that the time to diagnosis has not shortened over the years. This is despite better knowledge of the pathology of the disease and early detection programmes (12). The average time until a diagnosis is confirmed is 10.5 years for adults.

In childhood, acroparaesthesia (13), hypo-, anhidrosis $(14,15)$ and gastrointestinal complaints are particularly prominent (16). Angiokeratomas can also occur (14).

With increasing age, damage to the myocardial cells, the glomerula and the peripheral neurons and glia cells develops.

The consequences are progressive left ventricular hypertrophy (LVH), high-grade cardiac dysrhythmia, renal insufficiency, apoplexy, polyneuropathy, arthralgia, sensorineural hearing loss and unspecific gastro-intestinal complaints.

Until 2001 the disease could only be treated symptomatically. With the development of enzyme replacement therapy (ERT), a causal therapy became possible (17). Here, a biotechnologically produced alphagalactosidase is infused intravenously at two-week intervals. Two preparations are available, agalsidase alfa, produced from human cells and agalsidase beta, produced from the ovarian cells of the Chinese hamster.

Chaperone therapy is also available, which induces a correct folding of alpha-galactosidase. The preparation Migalastat (18) has been authorised in the European Union since 2016.

Lucerastat is a new drug that directly inhibits glucosylceramide synthase. The mechanism of action corresponds to a substrate reduction therapy. The drug is currently in phase III trials.

The ultimate cure, gene therapy, of FD is currently subject of many studies. This involves the use of DNA or RNA in a vector-usually a virus vector-with the code for the GLA gene (alpha-galactosidase gene). With viral vectors, however, there is a risk of side effects such as cancer through inactivation of tumour suppressor genes. It is also important to mention that the germ cells are not affected by gene therapy, thus, continue to carry the defective GLA gene, so that the inheritance of the disease is still not broken. Germ line therapies are prohibited in most countries on humans.

As early diagnosis is of the highest importance in FD, it is important to screen and diagnose the disease as early as possible. Once the diagnosis is made, the patient has to be closely monitored and treated bearing in mind the multitude of different organ involvements. This necessitates a multidisciplinary approach.

Treatment options and algorhythms are reviewed in the article of Oder $e t a l$. As the specificities of the heart are reviewed in dept by Linhart et al. in this issue, we focus on all organs other than the heart.

In this article we focus on the following questions:

* Who should we screen?

* How to prove the diagnosis?

* How to manage the patient?

We present the following article in accordance with the Narrative Review reporting checklist (available at http:// dx.doi.org/10.21037/cdt-20-845).

\section{Methods}

We searched for references relevant to the topic in Medline, personal libraries, discussion with experts in the field of reviews of the literature and personal experience participating in and writing several reviews of the literature.

\section{Screening}

Early diagnosis is prerequisite for the reduction of morbidity and mortality. Causes of death in Fabry patients are primarily cardiovascular events as renal function can be substituted by dialysis $(19,20)$.

As FD is a genetic disease, pedigree analysis and genetic counselling are an essential part of diagnostics. In families with index patients and/or high risk populations, a screening 
Table 1 Fabry diagnosis with the following organ manifestation of unclear etiology

\begin{tabular}{ll}
\hline Organ manifestation & Diagnosis \\
\hline Cardiac & Left ventricular hypertrophy $>12 \mathrm{~mm}$, high-grade rhythm disturbances \\
Renal & Younger patients with renal insufficiency of unknown etiology, microalbuminuria \\
CNS & stroke of unclear etiology \\
\hline
\end{tabular}

CNS, central nervous system.

should be carried out early on $(21,22)$.

Although genome analysis is the gold standard, it is not necessary to perform in all patients.

In men, due to the $\mathrm{x}$-linked chromosomal inheritance, a missing/significantly reduced alpha-galactosidase from leukocytes or plasma can be diagnosed in an enzyme assay. However, for completion, a mutation analysis should be performed.

In women, a molecular genetic examination in dry blood is necessary to ensure a reliable diagnosis.

While under therapy, the effects of treatment should be monitored.

The biomarker lyso-Gb3, the deacetylated form of Gb3 (plasma globotriaosylsphingosine) in EDTA or dry blood is available for the assessment of the course of the disease. LysoGb3 correlates with the stage of the disease, the detected mutation and can be used to monitor ERT (23). In patients with lyso-Gb3 $<1.3 \mathrm{nmol} / \mathrm{l}$ (standard value $<0.8 \mathrm{nmol} / \mathrm{L}$ ) without typical symptoms, a Fabry can be excluded. Furthermore, the measurement of Gb3 in urine and plasma is available. However, it should be noted that in the case of non-classical mutations, Gb3 concentrations in urine may be normal (24). Gb3 can also be detected in biopsies of the kidney, myocardium and skin.

Lyso-Gb3 levels are significantly higher in male patients with classic FD than in late-onset FD (25). In female FD patients, the lyso-Gb3 level is often within the normal range or slightly elevated (26). Elevated lyso-Gb3 levels are found in $40 \%$ to $60 \%$ of female FD patients (27).

A polymorphism in the GLA gene always shows normal lyso-Gb3 levels (11). However, lyso-Gb3 levels do not correlate with disease severity (28). Therefore, further biomarkers are needed. Furthermore, lyso-Gb3 is not a prognostic biomarker under ERT (29) or chaperone therapy with migalastat.

Although FD is a multifaceted disease, some patient group should specifically be addressed and screened for FD. These are patients with unclear LVH $>12 \mathrm{~mm}$, who may also have higher-grade dysrhythmia $(30,31)$, patients with renal insufficiency of unknown etiology associated with microalbuminuria (32) and younger patients (15-55 years) with stroke of unclear cause (33). Table 1 summarizes organ manifestations of unclear etiology. An algorithm by Vardarli (34) clearly shows the way of screening (Figure 1).

\section{Diagnosis}

FD is more than the disease of one organ. The disease affects almost all human organs albeit to varying degrees (Table 2). Thus, patients diagnosed with FD should be transferred to a centre. Cooperation between the various disciplines is essential to ensure optimal and individualized diagnosis and treatment.

Diagnostics in the centre include anamnesis with pedigree preparation, clinical examination, laboratory testing, genetic testing, and various imaging procedures (34-36).

\section{Kidney}

In up to $50 \%$ of male and $20 \%$ of female patients kidney function is impaired (4).

Thus, kidney evaluation is essential. Before the introduction of ERT, renal failure occurred in $42 \%$ of male patients (19).

The earliest diagnostic parameter is proteinuria, which is already detectable in adolescence (2).

If the proximal tubules damaged, isothenuria and polyuria may result (37).

In the kidney Gb3, the bioactive molecule accumulates in tubules, mesangium cells, podocytes, interstitium, vascular endothelium, and smooth muscle cells. Furthermore, in podocytes CD74 and TGF-1 expression is induced. CD74, a macrophage migration inhibitory factor (MIF) receptor involved in signal transduction, stimulates cytokines expression resulting in apoptosis and inflammation. TGF1 stimulates the production of extracellular matrix proteins such as fibronectin and collagen type IV particularly in the glomeruli but also in the interstitium (38). These processes result in the development of focal and global 


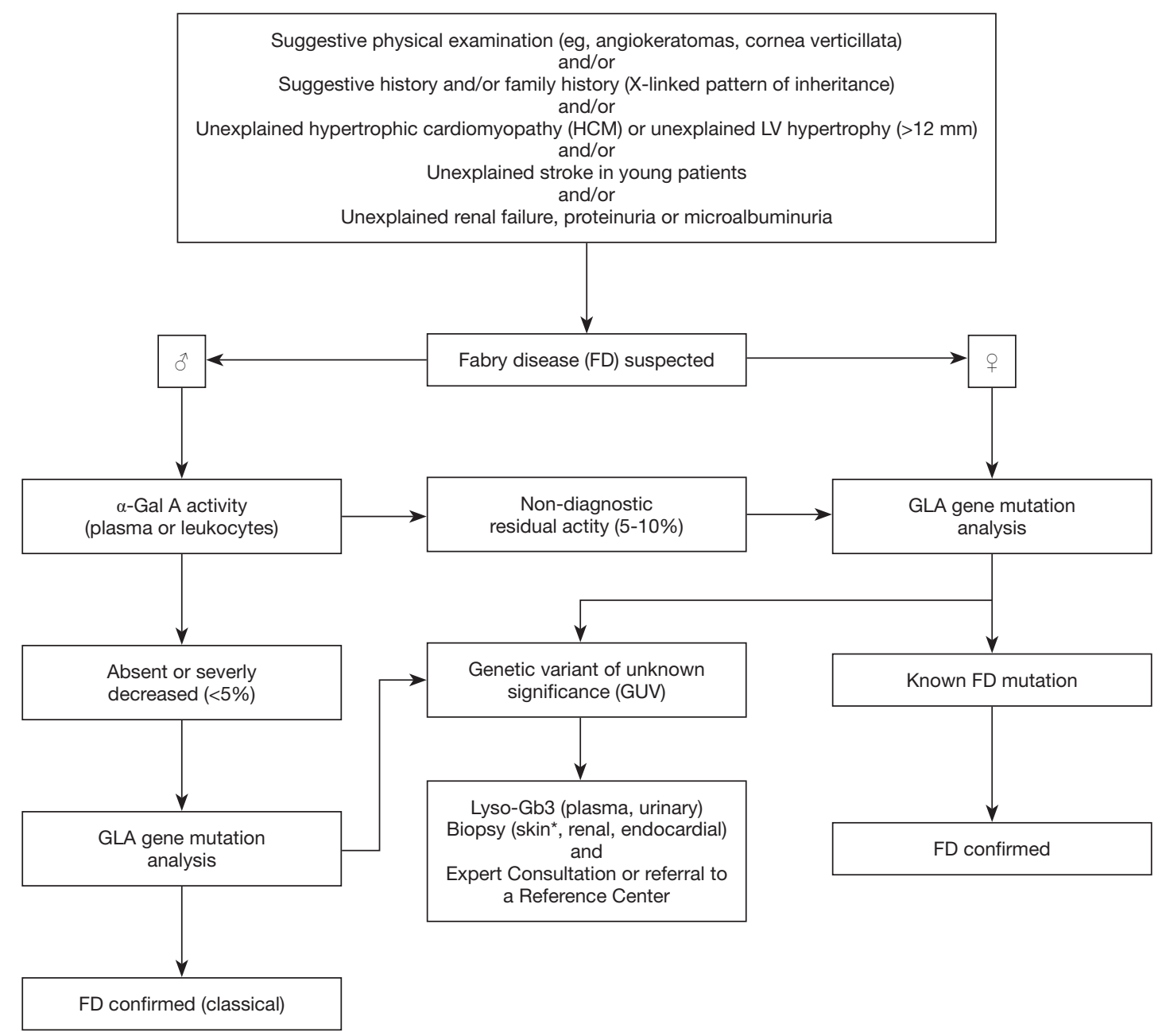

Figure 1 Updated diagnostic algorithm for FD. Adapted with permission from Vardarli I, Rischpler C, Herrmann K, et al. Diagnosis and Screening of Patients with Fabry Disease. Ther Clin Risk Manag 2020;16:551-8.

glomerulosclerosis with tubular atrophy, interstitial fibrosis and inflammation in the second decade of life (39) (Figure 2).

As a consequence eGFR annually decreases at a rate of -2.93 to $-6.8 \mathrm{~mL} / \mathrm{min} / 1.73 \mathrm{~m}^{2}$ in male, and -1.02 to $2.1 \mathrm{~mL} / \mathrm{min} / 1.73 \mathrm{~m}^{2}$ female patients. The prognosis is worse in the presence of proteinuria exceeding $>1 \mathrm{~g} /$ day (40).

To verify the diagnosis of FD in kidneys, a biopsy with electron microscopic detection of zebra bodies, an onionshell-like wound myelin in the cytoplasm of the podocytes, is the gold standard (41). A detection of Maltese crosses in the urine sediment is an indication of an FD, but also occurs with other glomerulonephritides. Another rather new option is the detection of CD77-positive epithelial cells in the urine with a sensitivity of $97.1 \%$ and a specificity of $100 \%$ for FD (42).
However, this has not been validated in larger studies so far.

\section{Cardiac}

Cardiac involvement is also very important, accounting for $40 \%$ to $60 \%$ of FD patients (43). The deposition of Gb3 in myocytes, vascular endothelial cells and valvular fibroblasts promotes $\mathrm{LVH}$ and consequential heart insufficiency. An electrocardiogram (ECG) is essential for the detection of arrhythmias as well as for initial diagnosis and followup of the LVH. Short PR interval, sinus bradycardia and atrioventricular conduction disturbances are possible $(44,45)$. Long-duration ECG is recommended to rule out higher grade arrhythmias (46). Echocardiography, and MRI are becoming increasingly important to specifically characterize LVH (22). Particularly during follow-up, high-sensitive 
Table 2 Organ manifestations and symptoms

\begin{tabular}{|c|c|}
\hline Organ manifestation & Symptoms \\
\hline Cardiac & Left heart hypertrophy, cardiac fibrosis, rhythm disturbances, sudden cardiac death \\
\hline CNS & TIA, stroke \\
\hline Peripheral nervous system & Small fiber neuropathy, hypohidrosis \\
\hline Gastrointestinal abdominal & Pain, diarrhoea alternating with constipation, nausea, vomiting \\
\hline Vascular disease & Increased intima-media thickness \\
\hline Dermatologic & Angiokeratomas \\
\hline Ophthalmologic & Cornea verticillate, conjunctival and retinal vasculopathy \\
\hline Lymphatic & Lymphedema \\
\hline Skeletal & Osteopenia, osteoporosis \\
\hline Face & Facial dysmorphism \\
\hline
\end{tabular}

CNS, central nervous system; ENT, ear, nose, throat.

Troponin T (hs-TNT) may be useful as a biomarker (47).

\section{Neurology}

The accumulation of Gb3 in the nerves induces a rather typical small-fibre neuropathy. Common signs are acroparaesthesia of the palms of hands feet (48). Triggers of possible pain crises are stress, fever, heat amongst other factors $(49,50)$.

The accumulation of $\mathrm{Gb} 3$ in the vasculature of the brain induces vasculopathy and dilated arteriopathy. These cerebrovascular complications can become apparent in the form of transient ischemic attacks (TIA) or strokes (51). The underlying accumulation of $\mathrm{Gb} 3$ can be detected as white matter lesions in the fluid-attenuated inversion recovery (FLAIR) sequence of magnetic resonance imaging (MRI). Furthermore, MRI can also be used to visualize other signs common in FD patients such as vascular abnormalities or a symmetrically high signal in the thalamus (pulvinar sign) in T1-weight (52).

\section{ENT (ear, nose, throat)}

The accumulation of glycosphingolipids causes a direct damage in the stria vascularis and ganglion cells as well as to the vasculature of the ear. The resulting vestibulo-chochlear disorder may lead to dizziness, tinnitus and sudden deafness, or an inner ear hearing loss with impaired sound sensation and disturbance in the high-frequency range (53). Cochlear damage frequently impairs sensorineural hearing of the inner ear $(54,55)$.

\section{Gastro-intestinal tract}

The cause of gastro-intestinal symptoms is probably a deposition of $\mathrm{Gb} 3$ in the ganglia autonomica of intestine and mesenteric blood vessels (16). The consequential abdominal pain, nausea, vomiting and diarrhea can already occur in childhood $(16,56)$. About one third of FD patients describes abdominal complaints (57). The diagnosis is based on the patient's symptoms. One possibility to clarify the significance of such complaints is the Rome III questionnaire, which has been validated for the differential diagnosis of functional gastrointestinal disorders (FGID). Alternatively, a biopsy can also be performed (58). However, while most biopsies react positive in the anti-Gb3 immunostaining, some mutation variants are not detectable (59).

\section{Lung}

In the lungs, glycosphingolipids are deposited in pneumocytes, muciparous goblet cells, bronchial ciliate 

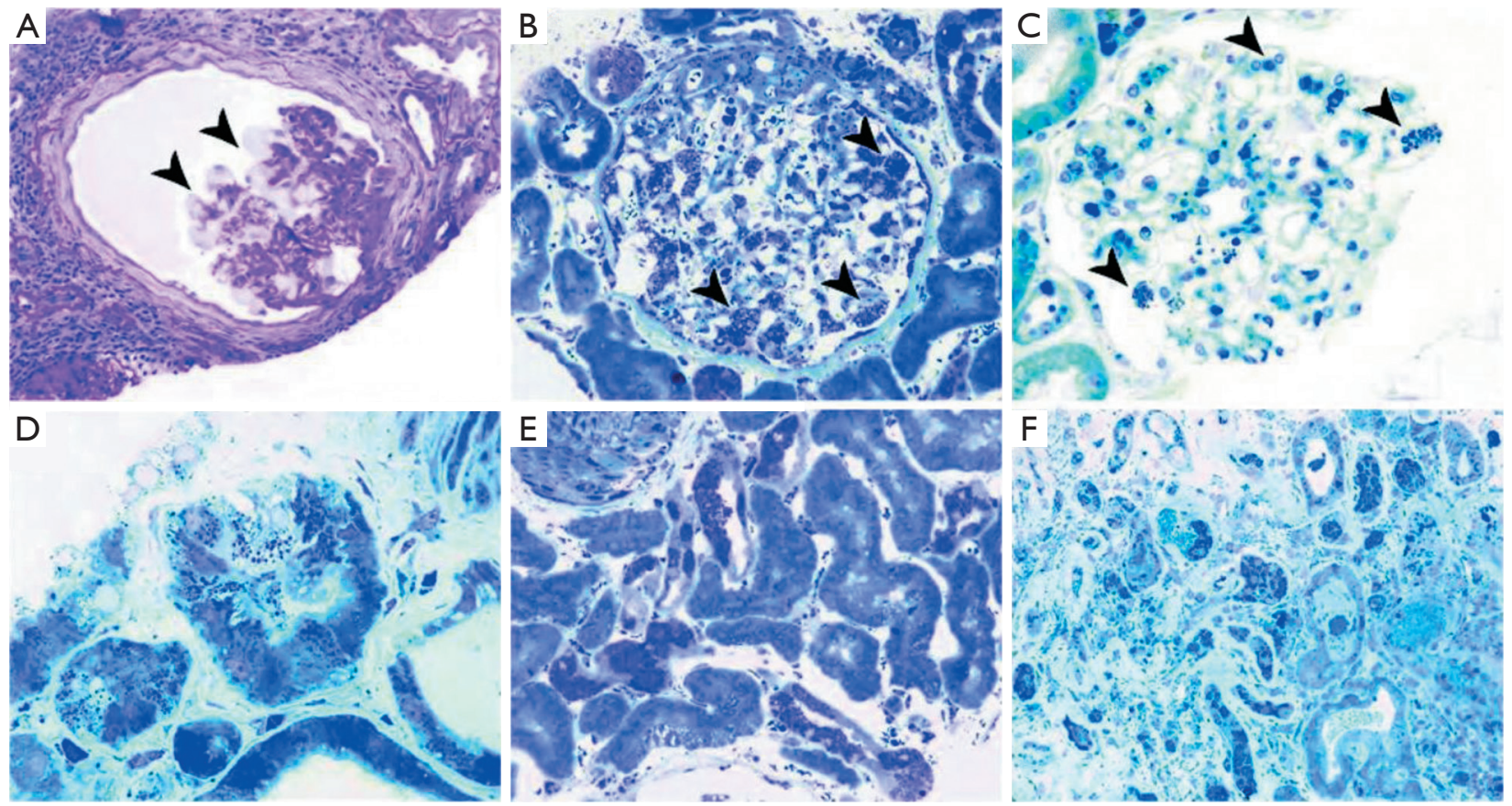

Figure 2 Scoring system for renal pathology in Fabry disease: report of the international study group of Fabry nephropathy (ISGFN). Vacuolization and glycosphingolipid deposits in Fabry nephropathy. (A) The glomerulus shows vacuolization of podocytes (arrowheads), and mild corrugation of glomerular basement membranes, with periglomerular and interstitial fibrosis (periodic acid-Schiff, $\times 400$ ). (B) The glomerulus shows massive expanded deposits in most podocytes (arrowheads) and also in mesangial and endothelial cells and parietal epithelial cells in this male Fabry patient (toluidine blue, $\times 400$ ). (C) The glomerulus shows occasional small deposits in podocytes (arrowheads) and rare deposits in mesangial areas in this female patient (toluidine blue, $\times 400$ ). (D) There are prominent deposits in some proximal tubular cells and peritubular capillary endothelium (toluidine blue, $\times 1,000$ ). (E) Numerous deposits in distal tubules and very rare deposits in proximal tubules. The vascular smooth muscle of a large artery (top left corner) also shows deposits, as do parietal epithelial cells lining the Bowman's capsule (bottom) (toluidine blue, $\times 1,000$ ). (F) Numerous deposits in distal tubules with rare deposits in proximal tubules and frequent deposits in peritubular capillaries and interstitium (toluidine blue, $\times 200$ ). Adapted with permission from Fogo AB, Bostad L, Svarstad E, et al. Scoring system for renal pathology in Fabry disease: report of the International Study Group of Fabry Nephropathy (ISGFN). Nephrol Dial Transplant 2010;25:2168-77.

epithelium, bronchial smooth muscle cells and pulmonary vessels (60). A narrowing of the bronchial system develops as a result of the deposit. The lung function test with measurement of the forced expiratory volume in the first second (FEV1) and the forced (expiratory) vital capacity (FEC) shows a deterioration in the course of the disease. This is aggravated by male patients, smoking, cardiac involvement due to FD and age at onset of ERT (61). Symptoms of pulmonary involvement are dyspnoea, dry cough and bronchospasm. they may also be present in cardiac involvement of FD and are therefore non-specific (62).

The prevalence of obstructive lung disease is higher in Fabry patients than in the general population $(18 \%$ and
$33 \%)(63)$. The diagnosis of obstructive lung disease is defined as FEV1/FEC < 70\% (64).

\section{Skin}

Damage based on the accumulation of Gb3 to the endothelium peri-vascular myocytes results in a dysregulation of the vasotonus of peripheral capillaries. The structural changes as well as inadequate vasodilatation lead to the appearance of reddish-livid, raised, punctiform, $1-5 \mathrm{~mm}$ in diameter efflorescences. Angiokeratomas (Figure 3), which can already appear in childhood, develop in $66 \%$ of male and $36 \%$ of female patients with FD (65). 


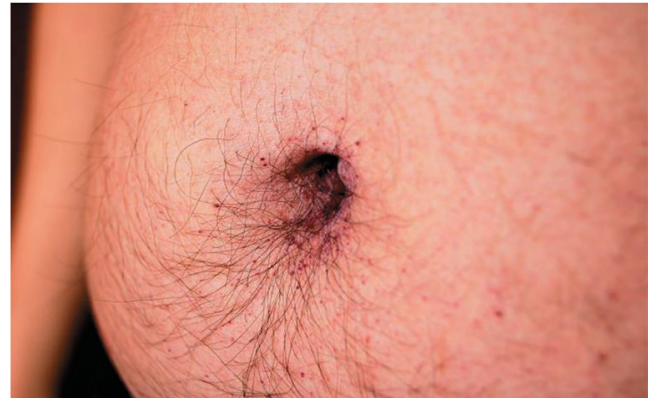

Figure 3 Picture of one of our patients with umbilical angiokeratomas.

Table 3 Organ systems and monitoring

\begin{tabular}{ll}
\hline Organ system & Monitoring \\
\hline Cardiac & Annually \\
Kidney & Every 6 months \\
Central nervous system & Every 3 years \\
Peripheral nervous system & Annually \\
ENT & As required \\
Gastrointestinal & If symptoms persist or worsen \\
Pulmonary & Every 2 years \\
\hline
\end{tabular}

\section{Eye}

Corneal opacity is an early symptom of FD and is known as cornea verticillata (66). It can be verified by Corneal opacity does not affect visual acuity. These changes have to be differentiated from other reasons for vortex keratopathy such as amiodarone or chloroquine (67).

\section{Management}

Multidisciplinarity is a prerequisite to ensure optimal care of patients with FD. FD patients should be cared for in a centre where the professional competence of the individual areas is reflected.

The reduction in life expectancy is determined by the degree of manifestation in kidneys, heart and central nervous system (68). Quality of life is also severely impaired by small-fibre polyneuropathy and hearing disorders. Regular follow-up examinations are therefore of great importance. Even in childhood, the quality of life can be reduced by chronic pain (69). Thus, regular evaluations via questionnaires is mandatory. The questionnaires available include SF-36, BPI (pain questionnaire), WHO5 (depression questionnaire) and MDI10. Table 3 summarizes recommended monitoring by organ system.

\section{Kidney}

Kidney parameters should be determined every 6 months. These include creatinine, at early stages cystatin C, urea, albuminuria ( $\mathrm{g}$ per $\mathrm{g}$ creatinine) and proteinuria ( $\mathrm{g}$ per $\mathrm{g}$ creatinine). Albuminuria and proteinuria should be measured in the morning spot urine, but can also be determined in 24-hour urine collections. Vitamin D levels should be measured during the winter months.

An ultrasound should be performed at least once at the initial examination. As a consequence of kidney deterioration, hypertension develop over time. Thus, a long-term blood pressure measurement is recommended annually (2).

\section{Cardiac}

ECG and echocardiography should be performed annually to assess arrhythmias and $\mathrm{LVH}$.

A 48-h Holter monitoring is also recommended annually for the detection of intermittent arrhythmias. Cardiac magnetic resonance imaging with gadolinium should be performed if progression is suspected or in intervals of $>2$ years (70).

\section{Neurology}

Initially doppler/duplex ultrasound to assess intima media thickening and ectasia of the basilar artery should be performed. An MRI of the brain should be performed approximately every 3 years in the case of central nervous involvement or in case of a stroke.

As pain is affecting many patients with FD, questionnaires for pain, depression, and over-all life quality (SF-36) should be performed on a yearly basis.

\section{Gastro-intestinal tract}

Abdominal sonography should be performed routinely at the initial presentation and at the time of the examination. Gastroscopy with biopsies and colonoscopy with biopsies should be performed only in case of unclear symptoms or as recommended for the standard population (71). 


\section{Lung}

If there are no other causes such as asthma, smoking, cardiac involvement by $\mathrm{FD}$, a lung function and radiograph are indicated (60). Deposition of Gb3 in bronchial, arteriolar and endothelial smooth muscle cells results in different imaging modalities (72).

\section{ENT}

At the initial examination, an ENT examination with hearing and possibly tinnitus diagnosis and examination of the vestibular apparatus is recommended (70,71).

As a follow-up, a sound threshold audiometry and, if necessary, tinnitus diagnostics and examination of the vestibular apparatus should be regularly carried out.

\section{Therapy}

\section{ERT}

Two preparations are currently available for causal therapy, ERT. Agalsidase alfa (Replagal $\left.{ }^{\circledR}\right)(73)$ and Agalsidase beta $\left(\right.$ Fabrazyme $^{\circledR}$ ). ERT is infused regularly at two-week intervals. Agalsidase alfa is given at $0.2 \mathrm{mg} / \mathrm{kg}$ body weight, agalsidase beta is given at $1.0 \mathrm{mg} / \mathrm{kg}$ body weight once every 2 weeks as an IV infusion (70). According to the consensus of the European Fabry working group, ERT should be started before the age of 18 in asymptomatic male patients with classic FD (30).

A new preparation, the pegunigalsidase alfa, a pegylated dimerized version of agalsidase alfa has an 80 -fold increase in half-life (74). Pegunigalsidase alfa has a reduced immunity, so it is assumed that a small amount of anti drug antibodies (ADA) are formed.

\section{Chaperon: Migalastat}

In addition, chaperone therapy with Migalastat has been available since 2016 . This can only be instilled if the mutation is predicted as "amenable". Migalastat induced a correct folding of alpha-galactosidase to increase enzyme activity (75). It is an oral therapy, the patient takes one tablet every 2 days.

\section{Substrate reduction therapy under investigation}

Lucerastat and Venglustat: It is an iminosugar. It inhibits the glucosylceramide synthase so that reduced glucosylceramides can be formed which are a precursor of the Gb3 $(76,77)$.

Gene therapy under investigation:

In ex vivo gene therapy, hematopoietic stem cells are collected from the patient and subjected to gene editing. the stem cells are then transfused back to the patient (78).

In the in vivo gene therapy, an infusion of adenoassociated virus (AAV) mediated gene transfer is carried out, resulting in increased enzyme activity levels (79).

\section{Conclusions}

$\mathrm{FD}$ is a rare $\mathrm{X}$-linked lysosomal storage disease. The deposition of Gb3 leads to multiple organ damage. Care in specialised centres with a multidisciplinary approach is therefore of great importance for the management of the disease.

\section{Acknowledgments}

Funding: None.

\section{Footnote}

Provenance and Peer Review: This article was commissioned by the Guest Editors (Yskert von Kodolitsch, Harald Kaemmerer, Koichiro Niwa) for the series "Current Management Aspects in Adult Congenital Heart Disease (ACHD): Part III" published in Cardiovascular Diagnosis and Therapy. The article has undergone external peer review.

Reporting Checklist: The authors have completed the Narrative Review reporting checklist. Available at http:// dx.doi.org/10.21037/cdt-20-845

Conflicts of Interest: All authors have completed the ICMJE uniform disclosure form (available at http:// dx.doi.org/10.21037/cdt-20-845). The series "Current Management Aspects in Adult Congenital Heart Disease (ACHD): Part III" was commissioned by the editorial office without any funding or sponsorship. MB reports personal fees from Vifor Pharma, outside the submitted work. The authors have no other conflicts of interest to declare.

Ethical Statement: The authors are accountable for all aspects of the work in ensuring that questions related to the accuracy or integrity of any part of the work are appropriately investigated and resolved. 
Open Access Statement: This is an Open Access article distributed in accordance with the Creative Commons Attribution-NonCommercial-NoDerivs 4.0 International License (CC BY-NC-ND 4.0), which permits the noncommercial replication and distribution of the article with the strict proviso that no changes or edits are made and the original work is properly cited (including links to both the formal publication through the relevant DOI and the license). See: https://creativecommons.org/licenses/by-nc-nd/4.0/.

\section{References}

1. Wilhelm Fabry (1560-1624)--the Other Fabricius. JAMA 1964;190:933.

2. Branton MH, Schiffmann R, Sabnis SG, et al. Natural history of Fabry renal disease: influence of alphagalactosidase A activity and genetic mutations on clinical course. Medicine (Baltimore) 2002;81:122-38.

3. Meikle PJ, Hopwood JJ, Clague AE, et al. Prevalence of lysosomal storage disorders. JAMA 1999;281:249-54.

4. Germain DP. Fabry disease. Orphanet J Rare Dis 2010;5:30.

5. Spada M, Pagliardini S, Yasuda M, et al. High incidence of later-onset fabry disease revealed by newborn screening. Am J Hum Genet 2006;79:31-40.

6. Hsu TR, Niu DM. Fabry disease: Review and experience during newborn screening. Trends Cardiovasc Med 2018;28:274-81.

7. Cairns T, Muntze J, Gernert J, et al. Hot topics in Fabry disease. Postgrad Med J 2018;94:709-13.

8. van der Tol L, Smid BE, Poorthuis BJ, et al. A systematic review on screening for Fabry disease: prevalence of individuals with genetic variants of unknown significance. J Med Genet 2014;51:1-9.

9. Desnick RJ, Astrin KH, Bishop DF. Fabry disease: molecular genetics of the inherited nephropathy. Adv Nephrol Necker Hosp 1989;18:113-27.

10. Arends M, Wanner C, Hughes D, et al. Characterization of Classical and Nonclassical Fabry Disease: A Multicenter Study. J Am Soc Nephrol 2017;28:1631-41.

11. Niemann M, Rolfs A, Stork S, et al. Gene mutations versus clinically relevant phenotypes: lyso-Gb3 defines Fabry disease. Circ Cardiovasc Genet 2014;7:8-16.

12. Reisin R, Perrin A, Garcia-Pavia P. Time delays in the diagnosis and treatment of Fabry disease. Int J Clin Pract 2017. doi: 10.1111/ijcp.12914.

13. Hopkin RJ, Bissler J, Banikazemi M, et al. Characterization of Fabry disease in 352 pediatric patients in the Fabry
Registry. Pediatr Res 2008;64:550-5.

14. Orteu CH, Jansen T, Lidove O, et al. Fabry disease and the skin: data from FOS, the Fabry outcome survey. Br J Dermatol 2007;157:331-7.

15. Gupta SN, Ries M, Murray GJ, et al. Skin-impedance in Fabry Disease: a prospective, controlled, non-randomized clinical study. BMC Neurol 2008;8:41.

16. Hoffmann B, Schwarz M, Mehta A, et al. Gastrointestinal symptoms in 342 patients with Fabry disease: prevalence and response to enzyme replacement therapy. Clin Gastroenterol Hepatol 2007;5:1447-53.

17. Lenders M, Brand E. Effects of Enzyme Replacement Therapy and Antidrug Antibodies in Patients with Fabry Disease. J Am Soc Nephrol 2018;29:2265-78.

18. McCafferty EH, Scott LJ. Migalastat: A Review in Fabry Disease. Drugs 2019;79:543-54.

19. Mehta A, Clarke JT, Giugliani R, et al. Natural course of Fabry disease: changing pattern of causes of death in FOS - Fabry Outcome Survey. J Med Genet 2009;46:548-52.

20. Waldek S, Patel MR, Banikazemi M, et al. Life expectancy and cause of death in males and females with Fabry disease: findings from the Fabry Registry. Genet Med 2009;11:790-6.

21. Sodré LSS, Huaira RMNH, Bastos MG, et al. Screening for Fabry Disease in Kidney Disease: a Cross-Sectional Study in Males and Females. Kidney Blood Press Res 2017;42:1258-65.

22. Hagège $A$, Réant $P$, Habib G, et al. Fabry disease in cardiology practice: Literature review and expert point of view. Arch Cardiovasc Dis 2019;112:278-87.

23. Aerts JM, Groener JE, Kuiper S, et al. Elevated globotriaosylsphingosine is a hallmark of Fabry disease. Proc Natl Acad Sci U S A 2008;105:2812-7.

24. Young E, Mills K, Morris P, et al. Is globotriaosylceramide a useful biomarker in Fabry disease? Acta Paediatr Suppl 2005;94:51-4; discussion 37-8.

25. Maruyama H, Miyata K, Mikame M, et al. Effectiveness of plasma lyso-Gb3 as a biomarker for selecting high-risk patients with Fabry disease from multispecialty clinics for genetic analysis. Genet Med 2019;21:44-52.

26. Smid BE, van der Tol L, Biegstraaten M, et al. Plasma globotriaosylsphingosine in relation to phenotypes of Fabry disease. J Med Genet 2015;52:262-8.

27. Duro G, Zizzo C, Cammarata G, et al. Mutations in the GLA Gene and LysoGb3: Is It Really Anderson-Fabry Disease? Int J Mol Sci 2018;19:3726.

28. Bichet DG, Aerts JM, Auray-Blais C, et al. Assessment of plasma lyso-Gb3 for clinical monitoring of treatment 
response in migalastat-treated patients with Fabry disease. Genet Med 2021;23:192-201.

29. Arends M, Biegstraaten M, Hughes DA, et al. Retrospective study of long-term outcomes of enzyme replacement therapy in Fabry disease: Analysis of prognostic factors. PLoS One 2017;12:e0182379.

30. Biegstraaten M, Arngrimsson R, Barbey F, et al. Recommendations for initiation and cessation of enzyme replacement therapy in patients with Fabry disease: the European Fabry Working Group consensus document. Orphanet J Rare Dis 2015;10:36.

31. Palecek T, Lubanda JC, Magage S, et al. [Cardiac manifestation of Fabry's disease: current knowledge]. Vnitr Lek 2004;50:846-51.

32. Branton M, Schiffmann R, Kopp JB. Natural history and treatment of renal involvement in Fabry disease. J Am Soc Nephrol 2002;13 Suppl 2:S139-43.

33. Bersano A, Borellini L, Motto C, et al. Molecular basis of young ischemic stroke. Curr Med Chem 2013;20:3818-39.

34. Vardarli I, Rischpler C, Herrmann K, et al. Diagnosis and Screening of Patients with Fabry Disease. Ther Clin Risk Manag 2020;16:551-8.

35. Smid BE, van der Tol L, Cecchi F, et al. Uncertain diagnosis of Fabry disease: consensus recommendation on diagnosis in adults with left ventricular hypertrophy and genetic variants of unknown significance. Int J Cardiol 2014;177:400-8.

36. Laney DA, Bennett RL, Clarke V, et al. Fabry disease practice guidelines: recommendations of the National Society of Genetic Counselors. J Genet Couns 2013;22:555-64.

37. Meroni M, Sessa A, Battini G, et al. Kidney involvement in Anderson-Fabry disease. Contrib Nephrol 1997;122:178-84.

38. Sanchez-Niño MD, Sanz AB, Carrasco S, et al. Globotriaosylsphingosine actions on human glomerular podocytes: implications for Fabry nephropathy. Nephrol Dial Transplant 2011;26:1797-802.

39. Fogo AB, Bostad L, Svarstad E, et al. Scoring system for renal pathology in Fabry disease: report of the International Study Group of Fabry Nephropathy (ISGFN). Nephrol Dial Transplant 2010;25:2168-77.

40. Schiffmann R, Warnock DG, Banikazemi M, et al. Fabry disease: progression of nephropathy, and prevalence of cardiac and cerebrovascular events before enzyme replacement therapy. Nephrol Dial Transplant 2009;24:2102-11.

41. Mahmud HM. Fabry's disease--a comprehensive review on pathogenesis, diagnosis and treatment. J Pak Med Assoc
2014;64:189-94.

42. Selvarajah M, Nicholls K, Hewitson TD, et al. Targeted urine microscopy in Anderson-Fabry disease: a cheap, sensitive and specific diagnostic technique. Nephrol Dial Transplant 2011;26:3195-202.

43. Akhtar MM, Elliott PM. Anderson-Fabry disease in heart failure. Biophys Rev 2018;10:1107-19.

44. Perry R, Shah R, Saiedi M, et al. The Role of Cardiac Imaging in the Diagnosis and Management of AndersonFabry Disease. JACC Cardiovasc Imaging 2019;12:1230-42.

45. Talbot AS, Lewis NT, Nicholls KM. Cardiovascular outcomes in Fabry disease are linked to severity of chronic kidney disease. Heart 2015;101:287-93.

46. Weidemann F, Maier SK, Stork S, et al. Usefulness of an Implantable Loop Recorder to Detect Clinically Relevant Arrhythmias in Patients With Advanced Fabry Cardiomyopathy. Am J Cardiol 2016;118:264-74.

47. Seydelmann N, Liu D, Kramer J, et al. High-Sensitivity Troponin: A Clinical Blood Biomarker for Staging Cardiomyopathy in Fabry Disease. J Am Heart Assoc 2016;5:e002839. Erratum in: J Am Heart Assoc 2016;5(9). pii: e002114.

48. Biegstraaten M, Hollak CE, Bakkers M, et al. Small fiber neuropathy in Fabry disease. Mol Genet Metab 2012;106:135-41.

49. Desnick RJ, Brady RO. Fabry disease in childhood. J Pediatr 2004;144:S20-6.

50. Schuller Y, Linthorst GE, Hollak CE, et al. Pain management strategies for neuropathic pain in Fabry disease--a systematic review. BMC Neurol 2016;16:25.

51. Moore DF, Scott LT, Gladwin MT, et al. Regional cerebral hyperperfusion and nitric oxide pathway dysregulation in Fabry disease: reversal by enzyme replacement therapy. Circulation 2001;104:1506-12.

52. Altarescu G, Moore DF, Pursley R, et al. Enhanced endothelium-dependent vasodilation in Fabry disease. Stroke 2001;32:1559-62.

53. Keilmann A, Hegemann S, Conti G, et al. Fabry disease and the ear. In: Mehta A, Beck M, Sunder-Plassmann G, editors. Fabry Disease: Perspectives from 5 Years of FOS. Oxford: 2006.

54. Schachern PA, Shea DA, Paparella MM, et al. Otologic histopathology of Fabry's disease. Ann Otol Rhinol Laryngol 1989;98:359-63.

55. Sergi B, Conti G, Paludetti G, et al. Inner ear involvement in Anderson-Fabry disease: long-term follow-up during enzyme replacement therapy. Acta Otorhinolaryngol Ital 2010;30:87-93. 
56. Sheth KJ, Werlin SL, Freeman ME, et al. Gastrointestinal structure and function in Fabry's disease. Am J Gastroenterol 1981;76:246-51.

57. Pensabene L, Sestito S, Nicoletti A, et al. Gastrointestinal Symptoms of Patients with Fabry Disease. Gastroenterol Res Pract 2016;2016:9712831.

58. O'Brien BD, Shnitka TK, McDougall R, et al. Pathophysiologic and ultrastructural basis for intestinal symptoms in Fabry's disease. Gastroenterology 1982;82:957-62.

59. Hilz MJ, Arbustini E, Dagna L, et al. Non-specific gastrointestinal features: Could it be Fabry disease? Dig Liver Dis 2018;50:429-37.

60. Faverio P, Stainer A, De Giacomi F, et al. Molecular Pathways and Respiratory Involvement in Lysosomal Storage Diseases. Int J Mol Sci 2019;20:327.

61. Franzen D, Haile SR, Kasper DC, et al. Pulmonary involvement in Fabry disease: effect of plasma globotriaosylsphingosine and time to initiation of enzyme replacement therapy. BMJ Open Respir Res 2018;5:e00277.

62. Svensson CK, Feldt-Rasmussen U, Backer V. Fabry disease, respiratory symptoms, and airway limitation - a systematic review. Eur Clin Respir J 2015. doi:10.3402/ ecrj.v2.26721.

63. Franzen D, Krayenbuehl PA, Lidove O, et al. Pulmonary involvement in Fabry disease: overview and perspectives. Eur J Intern Med 2013;24:707-13.

64. Pauwels RA, Buist AS, Ma P, et al. Global strategy for the diagnosis, management, and prevention of chronic obstructive pulmonary disease: National Heart, Lung, and Blood Institute and World Health Organization Global Initiative for Chronic Obstructive Lung Disease (GOLD): executive summary. Respir Care 2001;46:798-825.

65. Zampetti A, Orteu CH, Antuzzi D, et al. Angiokeratoma: decision-making aid for the diagnosis of Fabry disease. Br J Dermatol 2012;166:712-20.

66. Orssaud C, Dufier J, Germain D. Ocular manifestations in Fabry disease: a survey of 32 hemizygous male patients. Ophthalmic Genet 2003;24:129-39.

67. Moiseev SV, Ismailova DS, Moiseev AS, et al. Cornea verticillata in Fabry disease. Ter Arkh 2018;90:17-22.

68. Wanner C, Arad M, Baron R, et al. European expert consensus statement on therapeutic goals in Fabry disease. Mol Genet Metab 2018;124:189-203.

69. Ramaswami U, Whybra C, Parini R, et al. Clinical manifestations of Fabry disease in children: data from the
Fabry Outcome Survey. Acta Paediatr 2006;95:86-92.

70. Ortiz A, Germain DP, Desnick RJ, et al. Fabry disease revisited: Management and treatment recommendations for adult patients. Mol Genet Metab 2018;123:416-27.

71. Eng CM, Germain DP, Banikazemi M, et al. Fabry disease: guidelines for the evaluation and management of multiorgan system involvement. Genet Med 2006;8:539-48.

72. Wang RY, Abe JT, Cohen AH, et al. Enzyme replacement therapy stabilizes obstructive pulmonary Fabry disease associated with respiratory globotriaosylceramide storage. J Inherit Metab Dis 2008;31 Suppl 2:S369-74.

73. Germain DP, Boucly C, Carlier RY, et al. Enzyme replacement therapy of lysosomal storage diseases. Rev Med Interne 2010;31 Suppl 2:S279-91.

74. Schiffmann R, Goker-Alpan O, Holida M, et al. Pegunigalsidase alfa, a novel PEGylated enzyme replacement therapy for Fabry disease, provides sustained plasma concentrations and favorable pharmacodynamics: A 1-year Phase 1/2 clinical trial. J Inherit Metab Dis 2019;42:534-44.

75. Feriozzi S, Hughes DA. New drugs for the treatment of Anderson-Fabry disease. J Nephrol 2021;34:221-30.

76. Welford RWD, Muhlemann A, Garzotti M, et al. Glucosylceramide synthase inhibition with lucerastat lowers globotriaosylceramide and lysosome staining in cultured fibroblasts from Fabry patients with different mutation types. Hum Mol Genet 2018;27:3392-403.

77. Guérard N, Oder D, Nordbeck P, et al. Lucerastat, an Iminosugar for Substrate Reduction Therapy: Tolerability, Pharmacodynamics, and Pharmacokinetics in Patients With Fabry Disease on Enzyme Replacement. Clin Pharmacol Ther 2018;103:703-11.

78. Huang J, Khan A, Au BC, et al. Lentivector Iterations and Pre-Clinical Scale-Up/Toxicity Testing: Targeting Mobilized CD34(+) Cells for Correction of Fabry Disease. Mol Ther Methods Clin Dev 2017;5:241-58.

79. Felis A, Whitlow M, Kraus A, et al. Current and Investigational Therapeutics for Fabry Disease. Kidney Int Rep 2019;5:407-13.

Cite this article as: Regenbogen $\mathrm{C}$, Braunisch MC, Schmaderer C, Heemann U. Fabry disease: what the cardiologist should consider in non-cardiac screening, diagnosis, and management-narrative review. Cardiovasc Diagn Ther 2021;11(2):661-671. doi: 10.21037/cdt-20-845 